University of South Carolina

Scholar Commons

$10-1-1987$

\title{
Moment Method Analysis of the Electric Field Under EHV Transmission Lines
}

T. L. Simpson

University of South Carolina - Columbia

Charles W. Brice

University of South Carolina - Columbia, brice@engr.sc.edu

Follow this and additional works at: https://scholarcommons.sc.edu/elct_facpub

Part of the Electrical and Computer Engineering Commons

\section{Publication Info}

Published in IEEE Transactions on Power Delivery, Volume 2, 1987, pages 1264-1270.

http://ieeexplore.ieee.org/xpl/Recentlssue.jsp?punumber=61

(C) 1987 by IEEE

This Article is brought to you by the Electrical Engineering, Department of at Scholar Commons. It has been accepted for inclusion in Faculty Publications by an authorized administrator of Scholar Commons. For more information, please contact digres@mailbox.sc.edu. 
voltages at other locations, phase-to-phase overvoltages at transformer terminations, arrester duties, back-to-back switching currents, outrush currents for close in faults, and capacitor currents during transformer switching operations.

2) It is important to consider the case of a capacitor switch restrike when designing protection for the capacitor bank and other system equipment. This case is important in sizing arresters located near the bank. For back-to-back switching, the impact of high frequency and high magnitude currents should be considered (closing resistors do

3) Neutral current monitoring can be used for backup unbalance protection in a grounded capacitor bank. The selection of the secondary burden and overvoltage protection should include considerations for possible back-to-back switching currents.

4) A programmable controller provides a convenient means of implementing all of the switching control objectives automatically for EHV capacitor banks. Considerations include criteria for tripping and closing individual capacitor banks, overvoltage protection functions, closing sequences to minimize switch duty, and system operating restrictions.

5) Equipment performance, including switching devices, capacitors, protective relaying, and control functions, should be monitored carefully during the testing phase of the installation to assure proper operation.

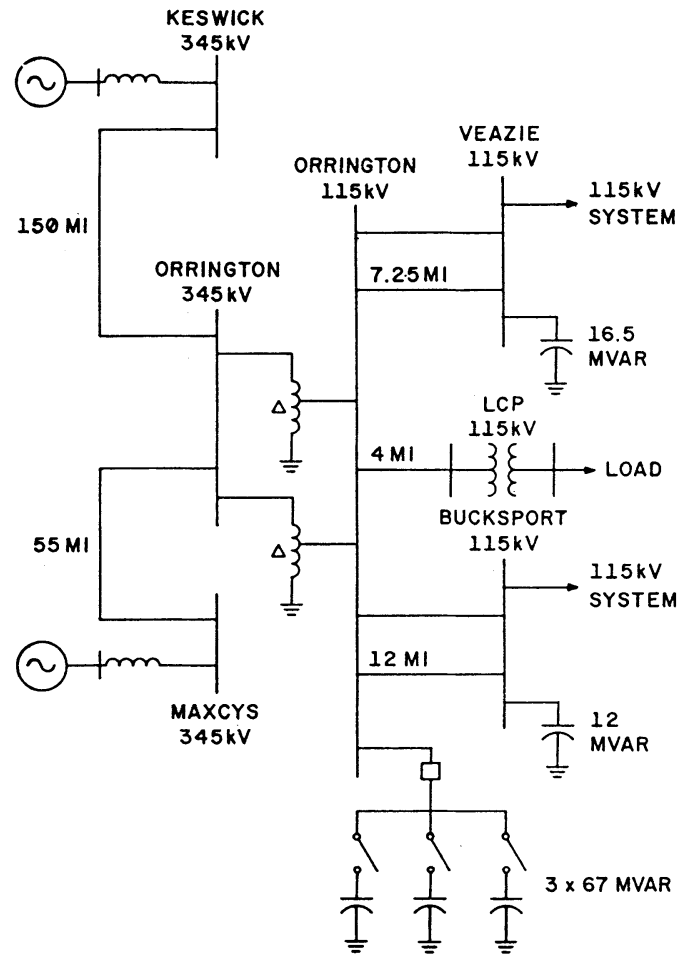

Fig. 1. One-line diagram illustrating capacitor application at Orrington $115 \mathrm{kV}$.

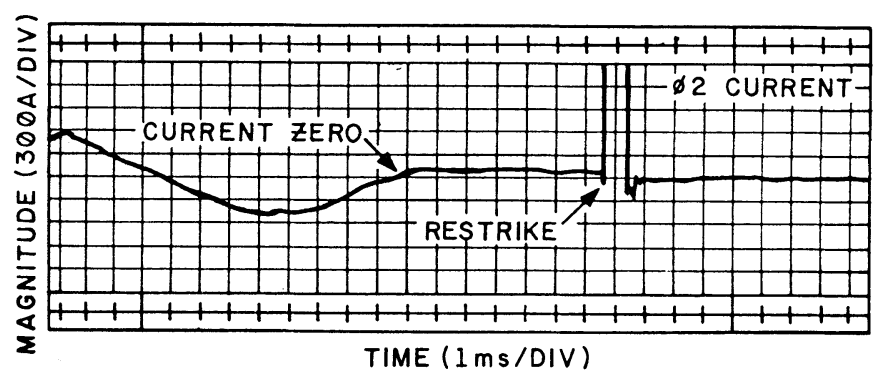

Fig. 2. Actual capacitor bank currents during a restrike.
86 T\&D 515-1

October 1987, pp. 1264-1270

\section{Moment Method Analysis of the Electric Field Under EHV}

\section{Transmission Lines}

\author{
T. L. Simpson, Senior Member, IEEE, and \\ C. W. Brice, III, Senior Member, IEEE \\ University of South Carolina, Columbia, SC
}

Concern for the possible effects of the fields near extrahigh-voltage (EHV) power transmission lines has resulted in an effort by the U.S. Department of Energy to define both the potential risk and the actual field environment as it presently exists under extra-high-voltage (EHV) lines. In particular, the effects of irregular terrain and vegetation have been observed experimentally in an extensive study by TVA at 2300 sites distributed over a wide variety of terrain types. Using a charge simulation method, the TVA researchers were able to determine the major influence of surface irregularity on the electric field in selected cases. However, the charge simulation method requires a very large array of discrete line sources placed judiciously below the surface. In this paper, a more convenient and economical method of field calculation is presented: the surface charge moment method.

Moment methods have been used extensively in the analysis of antennas and microstrip transmission lines. These methods overcome two limitations of the charge simulation method: 1) The surface charge can be modeled by a continuous distribution which resembles more accurately the physical picture. 2) Specified boundary values of the potential can be satisfied on the surface where the charge resides.

The paper contains the results of simulations, as well as a small number of comparisons to actual data. Statistical analysis of the results over the entire data set will be presented in a later paper. The simulations showed that there was no advantage to using the charge simulation method, since it was slower than the charge moment method for the same level of accuracy. This is primarily due to the fact that the charge moment method requires much fewer elements to simulate an irregular surface than does the charge simulation method.

For an example of the comparisons to actual data, consider site 20 on TVA's Johnsonville-Cordova line where the terrain profile is characterized by a slope of 0.3 percent and an $\mathrm{rms}$ surface deviation of $0.662 \mathrm{~m}$. Based on a mean slope of 7.3 percent, and a mean rms deviation of $0.538 \mathrm{~m}$ for the doublesided terrain profiles studied, this site exhibits a relatively low slope, but a high deviation. For this line, with a phase spacing of $12.2 \mathrm{~m}$ and a phase height of $14 \mathrm{~m}$, the electric field calculated with the Charge Moment Method agreed very well with the measured field. Here the rms residual error between measured and calculated fields was of 6 percent relative to the measured field. By contrast, the fields calculated with image theory, assuming a flat ground plane, for this case gave has a large rms residual error, 15 percent, because of the departure of the ground surface from a smooth plane.

Since intuition and experience are not required in the use of the moment method, it can be automatically applied to the analysis of a large body of data. Moment methods can be used to analyze three-dimensional surfaces; however, since the available experimental data was limited to two-dimensional ground surface profiles, this discussion is limited to the application of the charge moment method to a two-dimensional model of the $500-\mathrm{kV}$ transmission line typical of the TVA system.

For the calculation of the electric field under EHV transmission lines constructed over irregular terrain the charge moment method developed in this study proved to be both more accurate and more rapid than previously used techniques. Depending on the terrain, it was found that 20 to 30 strips were sufficient to accurately model the ground surface. 
Compared to the charge simulation method, which requires a much larger number of terms, this translates into a large reduction in the time required for matrix inversion and subsequent field calculation. In addition, the application of the charge moment method presented in the foregoing discussion was readily implemented in a data reduction program suitable for application to a large number of terrain profiles. By determining the major effects of irregular terrain on the electric field environment using the CMM model, it is expected that the effects of other, factors such as tree shielding, for example, may be identified more readily. Improved field prediction techniques should enable a more confident assessment of the field environment for future designs. system. This new area of analytical thought first called by Von Mises and Dodd "the asymptotic value," and later "la derniere valeur" by Gumbel, is the distribution of extremes. Furthermore, with his proposal, he takes into account the probable deviations caused by the sample size, which in "real life" is restricted by practical and economic factors. **

After demonstrating the practical advantages of using the extreme value theory in lieu of the normal curve method, the author briefly explains the basic steps for applying this technique, and lists the conclusions of this study.

* The probability that in any random sample we will find a given value $X$, that is, the largest or the smallest of all possible values, diminishes with increasing sample size.

\section{Use of Extreme Value Theory to Evaluate Effectiveness of Maintenance Programs in Electrical Distribution Systems}

\author{
Nicolas Estava M. \\ Grupo Baesa, Caracas, Venezuela
}

In this paper it is demonstrated that in evaluating the effectiveness of preventive maintenance programs, the electrical distribution manager benefits in speed of feedback information, accuracy of the system's true operational status, and ease of field data processing in the office when he uses the extreme value theory method in lieu of traditional normal curve, to calculate the system's sample period " $T$ " and the mean time between failures (MTBF) parameter.

The author uses ample outage data obtained from a distribution system located in southeastern Venezuela to calculate period " $T$ " and "MTBF," using first the normal curve model, and then the extreme value theory, applied to the same raw data. In calculating the MTBF, he uses the methodology of the normal curve with a $3 \sigma$ confidence level. For obtaining the sample size, or period " $\mathrm{T}$," he uses the normal-curve-based Kappa Square statistics, of Professor S. R. Calabro.

When the author makes the comparative analysis between the two techniques (read: normal curve versus extreme value theory), he emphasizes the fact that for same confidence levels, not only the system's sample time " $T$ "* reduced by almost 50 percent, but also those calculations done using the extreme value theory, are more reliable than those based on the more traditional method. The author attributes this evidence to the fact that the latter approach is a "distribution free statistics" that does not assume a priori that outage data will always fall within either the smooth symmetrical normal curve or any other mathematical model. Failure of an electrical distribution system to perform as expected ought to be treated as a "rare event," and the usual averages, modes, means, etc., used to characterize its operational behavior are not sufficient. The author advocates the introduction of a "new average," less constrained by biased assumptions and more compatible with the type of events occurring in the

* In this context, the sample period " $T$ " is the necessary time to observe the system outage frequency, after the maintenance program has been put into effect.
87 WM 161-3

October 1987, pp. 1276-1284

\section{Boundary Element Methods for Weakly Three Dimensional Quasi-Electrostatic Problems}

\author{
Robert G. Olsen, Senior Member, IEEE \\ Washington State University, Pullman, WA \\ Olov Einarsson, Member, IEEE \\ ASEA Research and Innovation, Västeràs, \\ Sweden
}

\begin{abstract}
Using perturbation theory, a Boundary Element Method is developed for solving a class of three dimensional electrostatic problems. This class includes problems for which an axially symmetric object is weakly coupled to other objects without symmetry so that the total geometry is three dimensional. A problem which falls into this class is that of a surge arrester located near one or more other arresters. The time required for set up and solution using this method is not significantly greater than that required for solving the axially symmetric problem by itself. A simple empirical formula for error is developed by comparing exact solutions with solutions developed using the method described here.
\end{abstract}

\section{Introduction}

Over the past ten years, computer programs for solving electrostatic problems have become an important tool for the design and analysis of high voltage components. More specifically they have been used to identify regions of high field strength where electrical breakdown might occur. The geometry near these regions is then redesigned to achieve a more uniform distribution of electric field and hence a lower probability of breakdown. In at least one computer program, this redesign process has been automated.

The most commonly used methods for solving these problems are the Finite Element Method (FEM), the Charge Simulation Method (CSM) and the Boundary Element Method (BEM). Other methods which are not as commonly used are the Finite Difference Method (FDM) and the Monte Carlo Method (MCM).

The purpose of this paper is to describe a technique based on the Boundary Element Method which is useful for an important class of three dimensional problems which will be defined here as "weakly three dimensional." Problems which fall into this category can be described with reference to Fig. 1 as follows. The region $(R)$ of interest contains an axially 